\title{
Direct Observation of IBW Induced Poloidal Flow in TFTR
}

\author{
B.P. LeBlanc, ${ }^{1}$ R.E. Bell, ${ }^{1}$ S. Bernabei, ${ }^{1}$ J.C. Hosea,${ }^{1}$ R. Majeski,${ }^{1}$ M. Ono, ${ }^{1}$ \\ C.K. Phillips, ${ }^{1}$ J.H. Rogers, ${ }^{2}$ G. Schilling, ${ }^{1}$ C.H. Skinner, ${ }^{1}$ J.R. Wilson ${ }^{1}$ \\ ${ }^{1}$ Princeton Plasma Physics Laboratory, Princeton, New Jersey, USA 08540 \\ ${ }^{2}$ Intevac, Inc, Santa Clara, California, USA 95054-2704
}

\begin{abstract}
Shearing of the plasma poloidal rotation velocity was observed during application of ion Bernstein wave power in the TFTR tokamak. The first evidence of corroboration between measured poloidal velocity shearing and actively induced Reynolds stress effects is presented. A model reproduces salient experimental features: The observed sheared flow occurs near the tritium fifth harmonic cyclotron resonance layer and depends strongly on the tritium density in agreement with the model. Furthermore, the model reproduces the observed insensitivity of the induced rotation to the tritium density in the region between the third deuterium harmonic layer and the fifth tritium harmonic layer.
\end{abstract}

The prospect of using externally driven waves to impel plasma motion capable of breaking up the turbulence eddies and thereby reduce transport[1] has renewed interest in Ion Bernstein Waves (IBW) [2] as a means to actively control transport. These waves are well suited for the task because of their short wavelength - on the order of the ion gyro radius - and strong single-pass localized absorption, when in proximity of an ion resonance. Following the encouraging results obtained on PBX-M [3] with externally launched IBW, an IBW experiment was planned and conducted on TFTR[4], during which sheared poloidal flow was 
indeed observed. In this letter, we analyze the experimental data and find it to be in agreement with a model proposed earlier [5], where the wave-induced Reynolds stress drives, in the present case, a spatially sheared poloidal rotation near the fifth tritium ion-cyclotron harmonic layer. Such results have important ramifications for active control of local transport in magnetic fusion devices.

The data presented here pertain to "direct launch" IBW, where we used mode transformation from edge-excited electron plasma waves into Ion Bernstein waves as a means to induce IBW in the plasma. For this purpose, a dedicated antenna, with RF current directed in the toroidal direction and comprising two pairs of current elements symmetrically distributed above and below the horizontal midplane was commissioned. Figure 1(a) shows a vertical cross-section of the plasma. The magnetic axis, $R_{o}$, is at $271 \mathrm{~cm}$, the third harmonic deuterium cyclotron resonance $\left(3 \Omega_{\mathrm{D}}\right)$ layer at $R_{3 \Omega \mathrm{D}} \approx 283 \mathrm{~cm}$, the fifth harmonic tritium $\left(5 \Omega_{\mathrm{T}}\right)$ layer at $R_{5 \Omega \mathrm{T}} \approx 314 \mathrm{~cm}$, and the plasma edge $R_{a}$ at $353 \mathrm{~cm}$. The antenna first surface is at $360 \mathrm{~cm}$. The antenna RF frequency was $\omega=76 \mathrm{MHz}$ and the toroidal field at the magnetic axis was $3.44 \mathrm{~T}$.

Selected experimental traces for a discharge with IBW are shown in Fig. 1(b). The bottom panel displays neutral beam and antenna launched powers, along with power radiated by the plasma. The 0.36 MW IBW power is added 1 second after the onset of the $2.5 \mathrm{MW}$ neutral beam injection (NBI). The top panel shows the perpendicular (diamagnetic) stored energy. The increase associated with IBW, although small compared to the effects of the neutral beam injection, is consistent with the relative power levels. The second panel displays the line integrated density, which also shows an increase during IBW. Although the NBI was deuterium only, the level of DT neutron emission shown in panel three is indicative of a sizeable tritium fraction among the plasma species. Spectroscopic measurements [6] point to the wall as the tritium source: about $35 \%$ of the Balmer-alpha recycling light is of tritium origin. Analysis of the data with TRANSP [7] has shown that a tritium ion density in the low $10^{12} \mathrm{~cm}^{-3}$ range had to be invoked to reproduce the DT neutron emission data. A preceding 
experiment with intensive tritium usage explains the wall condition. As we will see, the amount of tritium has important implications in the data analysis.

A poloidal velocity diagnostic [8] was installed during the last TFTR campaign. Its high throughput and opposing views permitted high precision measurements of the carbon poloidal velocity. Since we are interested in the change in poloidal velocity, $\Delta v_{\theta}$, occurring during IBW coupling, we used the following procedure: (1) the data was averaged over the 0.5 sec immediately preceding the application of IBW power; this averaging window occurred after the transient effects associated with the NBI onset had damped away, (2) this averaged profile was then subtracted over the whole time sequence. This procedure has the advantage of removing systematic effects between adjacent spatial channels. We will refer to $\Delta v_{\theta}$ as the flow. The data can be seen in Fig. 2(a), where we overlay the $\Delta v_{\theta}$ time evolution at six major radii. Positive $\Delta v_{\theta}$ corresponds to the ion-diamagnetic drift direction. It can be seen that, for times prior to the IBW application, $\Delta v_{\theta} \approx 0$ with a stable profile, as indicated by little change in the radial direction and a slow negative drift with time. On the other hand, when IBW was applied, rapid and substantial changes occurred, leading to a sheared $\Delta v_{\theta}$ profile. These modifications are landmarked by the ion cyclotron layers present in the plasma. Looking at Fig. 2(a), we can see that while $\Delta v_{\theta}$ fell to negative values in the $R_{3 \Omega \mathrm{D}}<R<R_{5 \Omega \mathrm{T}}$ region, it actually grew positive in the $R_{5 \Omega \mathrm{T}}$ vicinity, where the peak value occurs. In comparison, we can see in Fig. 2(b) results obtained when this same analysis procedure is applied to a reference "no-IBW" discharge. In this case, there is only a small change in the $\Delta \mathrm{v}_{\theta}$ profile, consistent with the slow drift mentioned above.

The presence of a negative flow in the $R_{3 \Omega \mathrm{D}}<R<R_{5 \Omega \mathrm{T}}$ region is a persistent feature of the experimental data, although one should keep in mind that the database for these conditions comprises only a handful of discharges. On the other hand, the driven flow in $R_{5 \Omega \mathrm{T}}$ vicinity appears to depend on the tritium concentration. Figure 3 illustrates this effect, where we 
overlay temporal evolution of $\Delta v_{\theta}$ data from three discharges. Discharges labeled "IBW A" and "no-IBW" correspond to the two cases presented above. Discharge "IBW B" had a significantly lower tritium density than IBW A; estimates of the tritium density levels follow. Looking at Fig. 3(a), we see that between the $3 \Omega_{\mathrm{D}}$ and $5 \Omega_{\mathrm{T}}$ layers, at $R \approx 295 \mathrm{~cm}$, both IBW A and IBW B discharges have a similar behavior, namely that of a negative poloidal flow compared to the no-IBW case. On the other hand IBW A and IBW B behave differently at $R=$ $312 \mathrm{~cm}$, near the $5 \Omega_{\mathrm{T}}$ layer: While IBW A shows a positive flow reaching $1 \mathrm{~km} / \mathrm{s}$, IBW B features a negative flow, falling to $-0.8 \mathrm{~km} / \mathrm{s}$.

Quantitative statements can be made about the tritium density, $n_{T}$, in plasmas IBW A and IBW B. In these discharges, where the NBI is done with deuterium, the strength of the neutron emission generated by the DT nuclear reactions is sensitive to the level of the tritium ion density in the core region of the target plasma. Figure 4(a) gives the temporal evolution of the experimental DT neutron production: the DT neutron rate for IBW A was $40 \%$ higher than for IBW B. We made use of the DT neutron data and of the edge recycling measurement mentioned above to provide constraints for the core and edge tritium density in TRANSP analyses, and extract $n_{T}$ profiles. In Fig. 4(b), we overlay $n_{T}$ obtained from TRANSP for these two discharges at time $t \approx 3.9-4.0 \mathrm{~s}$ : Near the $5 \Omega_{\mathrm{T}}$ layer location, $n_{T}$ for IBW A exceeded that of IBW B by $50 \%$. The change in $n_{T}$ was caused by the progressive wall cleaning during successive discharges; IBW B occurred four discharges after IBW A.

A ray-tracing code (IBWTRACER) has been developed to analyze the data. The original ray-tracing package, written by Ono [9], has been upgraded to multi-species profiles extracted from TRANSP analyses [10], and models the kinetic resonance structure as described below. We typically use five or six ion species including $\mathrm{H}, \mathrm{D}, \mathrm{T}$, a carbon-like impurity, and fast ions during NBI. Each species has its own density profile. The thermal ions are all assumed to have the same temperature profile. After computing the dispersion relation, fans of rays typically 40 rays are used - with a spectrum characteristic of the antenna are launched. The 
IBW power deposition is then computed when tracing rays. A subroutine of the ray-tracing software calculates the induced poloidal velocity $\Delta v_{\theta}$ using equation:

$$
\rho\left\langle\widetilde{v} \cdot \nabla \widetilde{v}_{\theta}\right\rangle+\mu_{n e o} \times \Delta v_{\theta}=
$$

where the first term of the LHS corresponds to the Reynolds stress induced by the IBW [5] and $\mu_{\text {neo }}$ is the neoclassical viscosity. For these calculations, we used a $\mu_{\text {neo }}$ formulation by Chang [11]. The second term of the LHS is usually written with brackets around $\Delta v_{\theta}$, but these were left out to remain consistent with the above notation where $\Delta v_{\theta}$ is an averaged quantity.

We used IBWTRACER to investigate the theoretical dependence of $\Delta v_{\theta}$ on $n_{T}$. Figure 5 presents predictions obtained for $n_{T}$ ranging from $0.4 \times 10^{12}$ to $2.0 \times 10^{12} \mathrm{~cm}^{-3}$ near the $5 \Omega_{\mathrm{T}}$ layer. In Fig. 5(a), we see that as $n_{T}$ increases, the dispersion develops a localized gradient around the $5 \Omega_{\mathrm{T}}$ resonance layer. In order to treat the $5 \Omega_{\mathrm{T}}$ resonance in a numerically tractable yet accurate manner, the Z-function approximation used in IBWTRACER to compute the real part of the dispersion relation was supplemented in order to achieve proper asymptotic behavior for $\left|\delta \omega / k_{\|}\right|<<v_{T}$ and for $\left|\delta \omega / k_{\|}\right|>>v_{T}$, where $\delta \omega=\omega-5 \Omega_{\mathrm{T}}, k_{\|}$is the parallel wave number and $v_{T}$ is the tritium thermal velocity. The usual kinetic damping terms with collisional modifications were used for the imaginary part [9]. In Fig. 5(b), we graph the predicted $\Delta v_{\theta}$ for carbon. The predicted driven flow is negative and insensitive to $n_{T}$ at $R \cong 295 \mathrm{~cm}$, as observed in Fig. 3(a). On the other hand, the predicted $\Delta v_{\theta}$ in the vicinity of the $5 \Omega_{\mathrm{T}}$ layer, $R \approx 314 \mathrm{~cm}$, varies strongly with $n_{T}$, from negative to positive values, in accordance with the experimental observation. For discharge IBW A where $n_{T} \cong 1.4 \times 10^{12} \mathrm{~cm}^{-3}$ one predicts a positive flow around $0.7 \mathrm{~km} / \mathrm{s}$, while for IBW B where $n_{T} \cong 0.9 \times 10^{12} \mathrm{~cm}^{-3}$ one gets a negative flow at -1.3 $\mathrm{km} / \mathrm{sec}$. The results, computed for time $t \cong 3.9-4.0 \mathrm{~s}$, are compared in Table I for discharges IBW A and IBW B. It is noteworthy that these calculations, while not including spatial 
poloidal momentum diffusive effects, are nevertheless able to reproduce correctly the sign of $\Delta v_{\theta}$ and to match the experimental data within roughly $50 \%$.

The IBW ray-tracing model provides satisfactory agreement with salient features of the experimental data. Firstly, the model explains the existence and $n_{T}$ dependency of the observed $\Delta v_{\theta}$ near the $5 \Omega_{\mathrm{T}}$ layer. Secondly, the model reproduces the negative $\Delta v_{\theta}$ observed in the $5 \Omega_{\mathrm{T}}<\omega<3 \Omega_{\mathrm{D}}$ region and explains its insensitivity to changes in the tritium density. The present analysis provides the first evidence of corroboration between measured poloidal velocity shearing and induced Reynolds stress. The ability to reproduce the $\Delta v_{\theta}$ behavior in space and with different tritium density gives credence to the model, and supports IBW-induced Reynolds stress as the origin of the poloidal drive observed on TFTR.

This work was supported by the U.S. Department of Energy Contract No. DE-AC02-76CHO-3073.

1 C.G. Craddock and P.H. Diamond, Phys. Rev. Lett. 67, 1536 1991)

2 Biglari, et al., RF Power in Plasmas, $9^{\text {th }}$ Topical Conf., AIP Conf. Proc. 244, p.376 (1991)

3 B.P. LeBlanc et al., Phys. Plasmas, 2, 741 (1995)

4 J.R. Wilson et al., Phys. Plasmas, 5, 1721 (1998)

5 M. Ono, et al., IAEA-CN-60/A-3-I-7, Seville, Spain, Oct. 1994

6 C. H. Skinner, et al., Nucl. Fus. 35, 143-151, (1995).

7 J. Ongena, M. Evrard, D. McCune, "Numerical Transport Codes", Transactions of Fusion Technology, March, 1998, Vol. 33, No. 2T, pp. 181-191.

8 R.E. Bell, Rev. Sci. Instrum. 68, 1273 (1997), and TFTR Poloidal Rotation Diagnostic R. E. Bell et al. Rev. Sci. Instrum. (to be published)

9 M. Ono, Phys. Fluids B, 5, 241 (1993)

10 B.P. LeBlanc, Proc. of the $12^{\text {th }}$ Top. Conf. On RF Power in Plasmas, AIP 403, p. 73 (1997) 
11 C.S. Chang, Phys. Fluids B 5, 4360 (1993) 


\begin{tabular}{|c|c|c|c|c|}
\hline \multirow{2}{*}{ Discharge } & \multicolumn{2}{|c|}{$\begin{array}{c}\text { IBW A } \\
\left(\text { high } n_{T}\right)\end{array}$} & \multicolumn{2}{c|}{$\begin{array}{c}\text { IBW B } \\
\left(\text { low } n_{T}\right)\end{array}$} \\
\hline$R(\mathrm{~cm})$ & $\begin{array}{c}295 \\
\left(R_{3 \Omega \mathrm{D}}<R<R_{5 \Omega \mathrm{T}}\right)\end{array}$ & $\begin{array}{c}314 \\
\left(R \approx R_{5 \Omega \mathrm{T}}\right)\end{array}$ & $\begin{array}{c}295 \\
\left(R_{3 \Omega \mathrm{D}}<R<R_{5 \mathrm{~W}}\right)\end{array}$ & $\begin{array}{c}314 \\
\left(R \approx R_{5 \Omega \mathrm{T}}\right)\end{array}$ \\
\hline $\begin{array}{c}\Delta v_{\theta}(\mathrm{km} / \mathrm{s}) \\
\text { Experimental }\end{array}$ & $-2.1 \pm 0.1$ & $+0.7 \pm 0.1$ & $-2.1 \pm 0.1$ & $-0.8 \pm 0.1$ \\
\hline $\begin{array}{c}\Delta v_{\theta}(\mathrm{km} / \mathrm{s}) \\
\text { IBWTRACER }\end{array}$ & -1.25 & +0.6 & -1.25 & -1.3 \\
\hline
\end{tabular}

Table I: Comparison of computed poloidal driven flow with measurements. 


\section{Figure Captions:}

Fig. 1: (a) Vertical cross-section showing resonance layer $3 \Omega_{\mathrm{T}}$ and $5 \Omega_{\mathrm{T}}$ locations, magnetic axis $R_{o}$ and outer plasma edge $R_{a}$. The trajectories of four rays onto the poloidal plane are also shown. (b) Selected discharges time traces: stored energy, line-integrated density, DT neutrons, heating and radiated powers.

Fig. 2: Experimental carbon $\Delta v_{\theta}$ at six major radius locations during (a) discharge with IBW power; (b) discharge without IBW power.

Fig. 3: Overlay of $\Delta v_{\theta}$ time evolution for IBW discharges IBW A (higher tritium density) and IBW B (lower tritium density), and for a no-IBW discharge: (a) at $R=295 \mathrm{~cm}$, (b) at $R=$ $312 \mathrm{~cm}$.

Fig. 4: Comparison between discharges IBW A and IBW B: (a) DT neutron source strength temporal evolutions; (b) TRANSP evaluation of $n_{T}$ at $t \cong 4 \mathrm{~s}$.

Fig. 5: IBWTRACER predictions over $n_{T}$ range $0.4-2.0 \times 10^{12} \mathrm{~cm}^{-3}$ : (a) real part of the dispersion relation; (b) IBW poloidal drive $\Delta v_{\theta}$. 


\section{TOP}

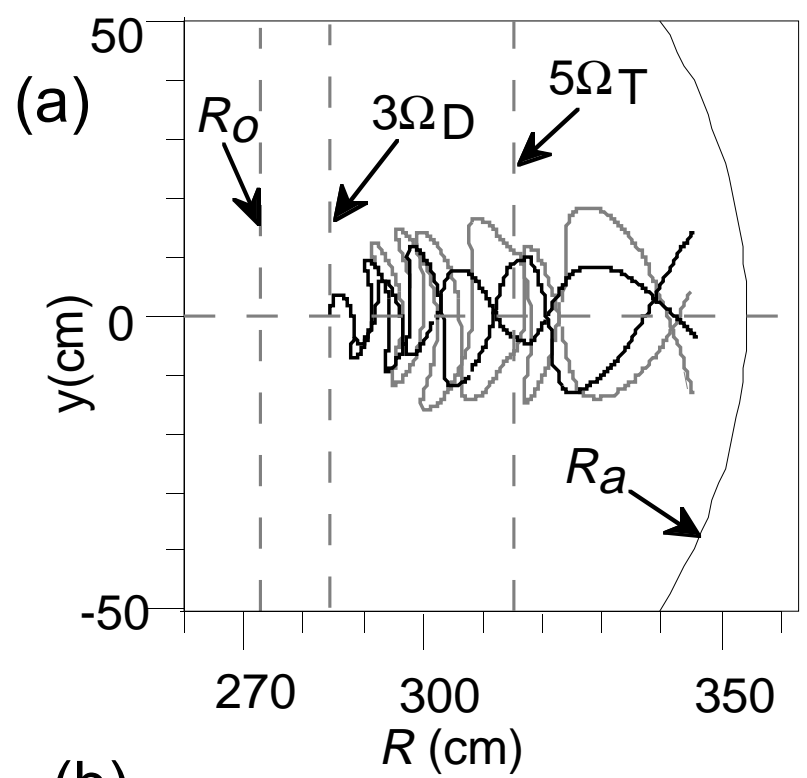

(b)
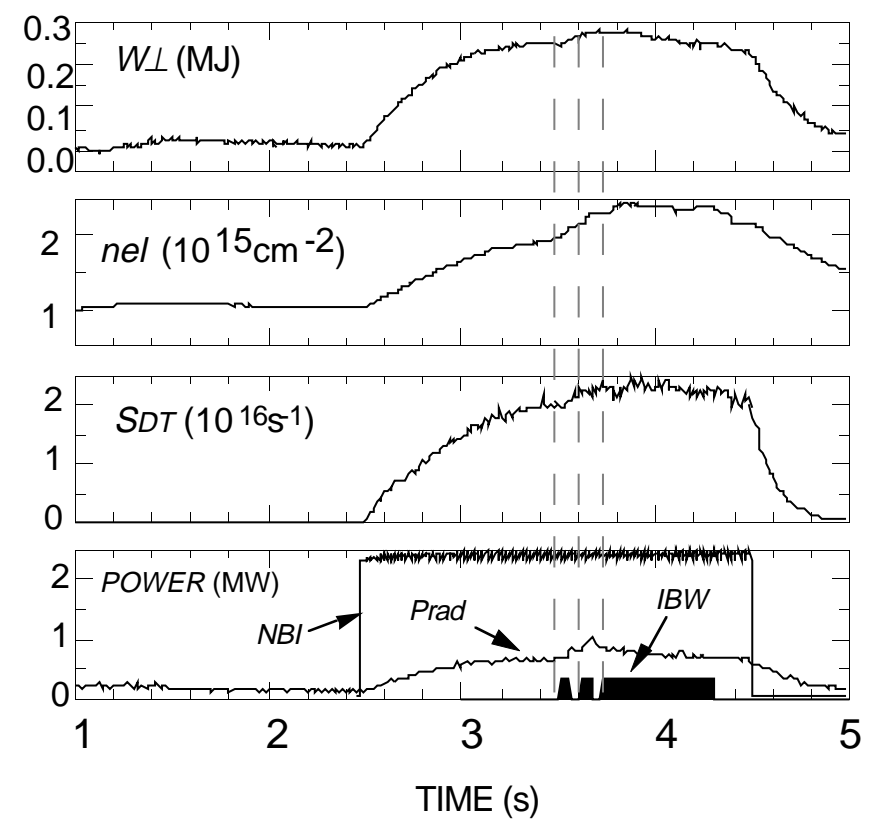

Fig.1, LeBlanc et al., Phys. Rev. Lett. 


\section{TOP}
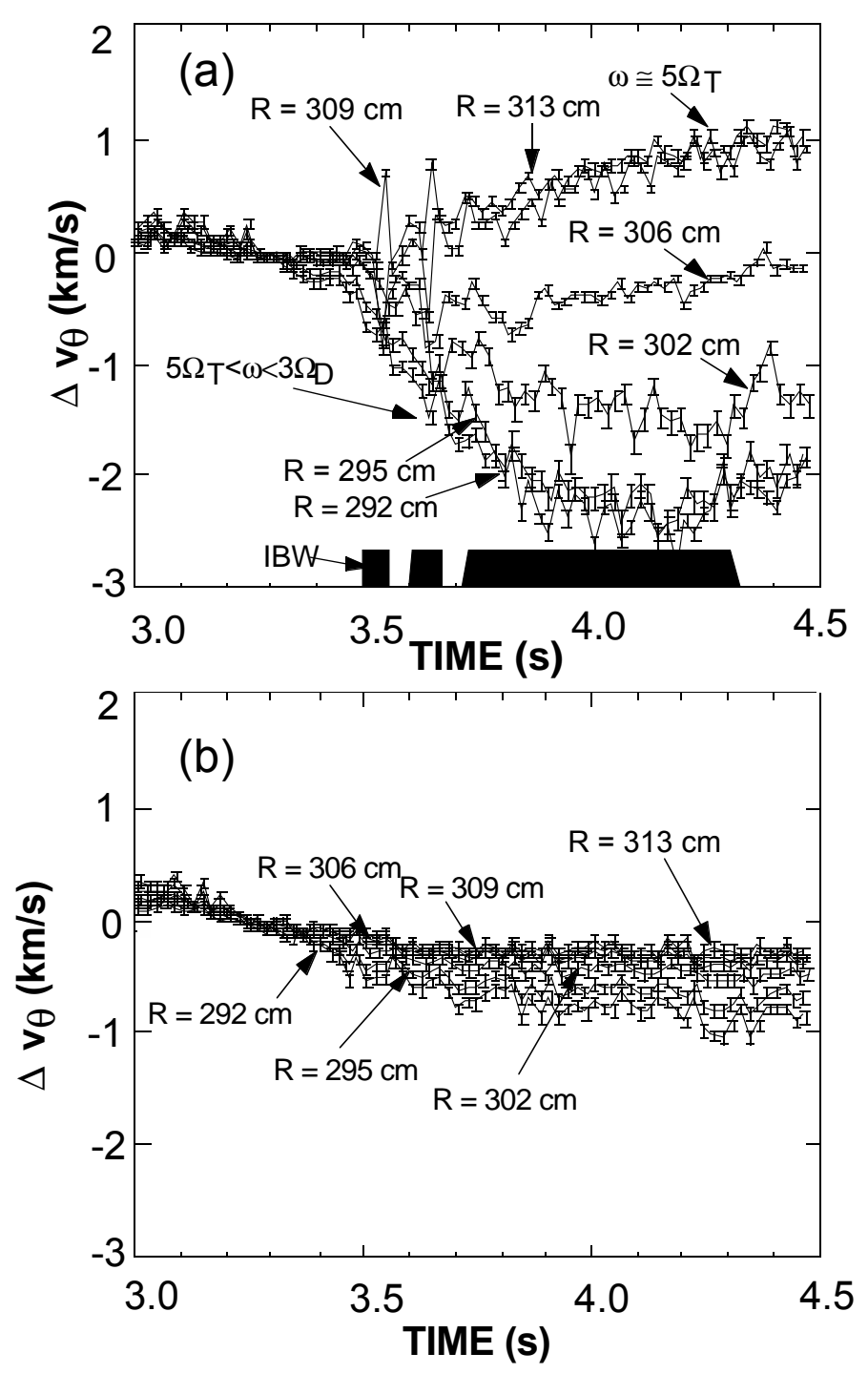

Fig. 2, LeBlanc et al., Phys. Rev. Lett. 

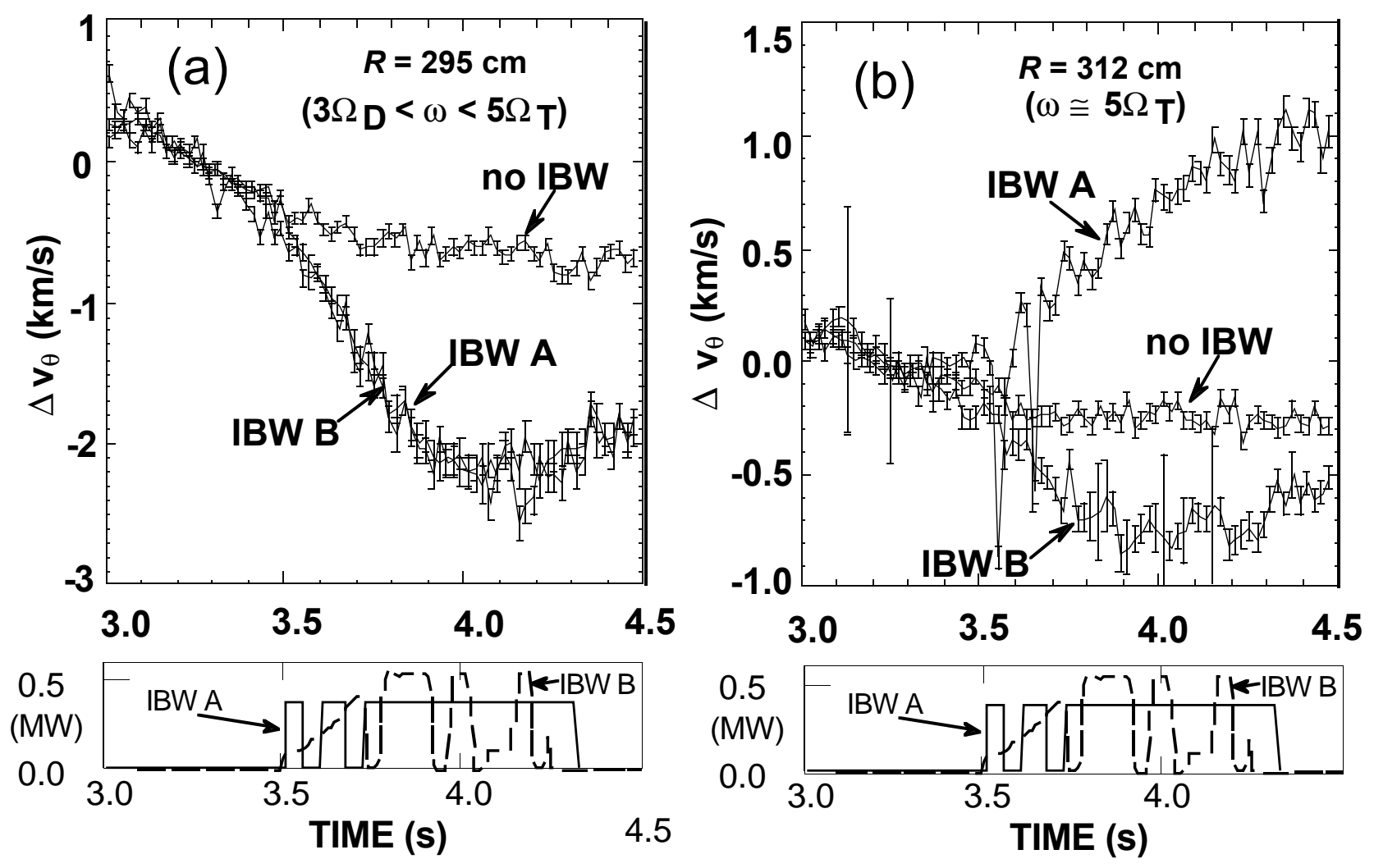

Fig.3, LeBlanc et al., Phys. Rev. Lett. 

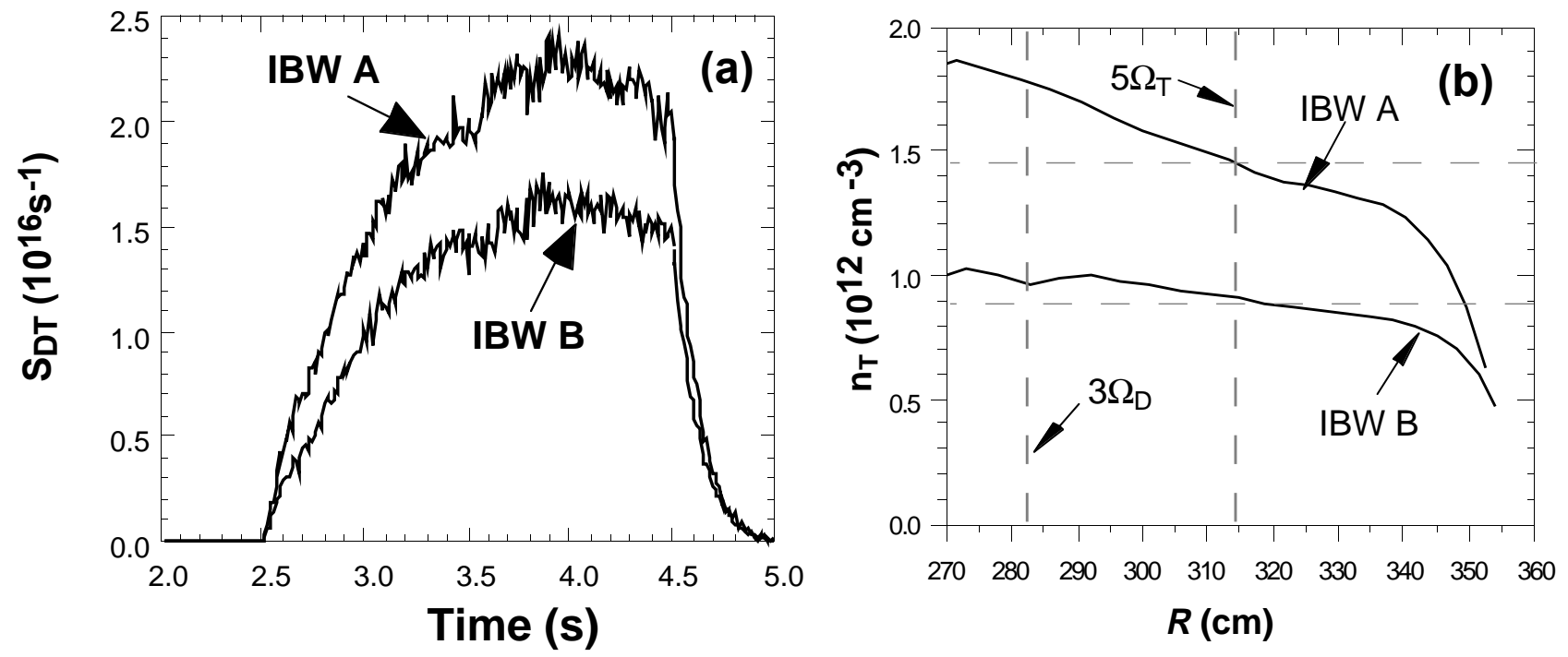

Fig.4, LeBlanc et al., Phys. Rev. Lett. 

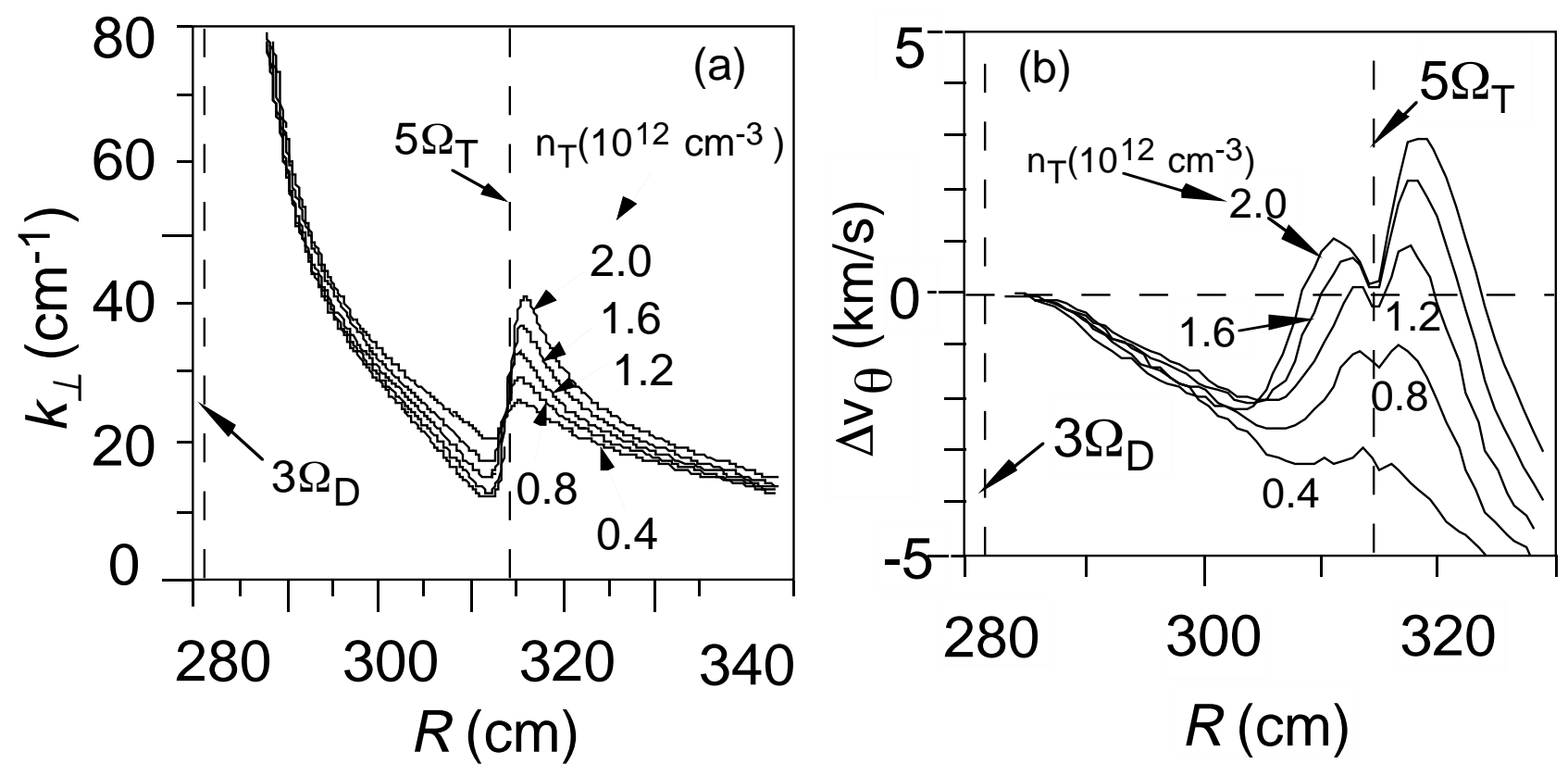

Fig. 5, LeBlanc et al., Phys. Rev. Lett. 